\title{
Changing trends in liver cancer incidence by race/ethnicity and sex in the US: 1992-2016
}

\author{
Maxwell Salvatore ${ }^{1}\left(\mathbb{D} \cdot\right.$ Jihyoun Jeon $^{1}$ (i) $\cdot$ Rafael Meza $^{1} \mathbb{C}$
}

Received: 23 September 2018 / Accepted: 1 October 2019 / Published online: 12 October 2019

(c) The Author(s) 2019

\begin{abstract}
Purpose Liver cancer incidence continues to increase while incidence of most other cancers is decreasing. We analyze recent and long-term trends of US liver cancer incidence by race/ethnicity and sex to best understand where to focus preventive efforts.

Methods Liver cancer incidence rates from 1992 to 2016 were obtained from the Surveillance, Epidemiology, and End Results registry. Delay-adjusted age-standardized incidence trends by race/ethnicity and sex were analyzed using joinpoint regression. Age-specific incidence was analyzed using age-period-cohort models. Hepatitis C seroprevalence by cohort was calculated using National Health and Nutrition Examination Survey data.

Results Liver cancer incidence has peaked in males and Asian or Pacific Islanders. Hispanic males, a high-incidence population, are experiencing a decrease in incidence, although not yet statistically significant. In contrast, incidence continues to increase in females, although at lower rates than in the 1990s, and American Indian/Alaska Natives (AI/ANs). Liver cancer incidence continues to be higher in males. Non-Hispanic Whites have the lowest incidence among racial/ethnic groups. Trends largely reflect differences in incidence by birth-cohort, which increased considerably, particularly in males, for those born around the 1950s, and continues to increase in females and AI/ANs. The patterns in males are likely driven by cohort variations in Hepatitis $\mathrm{C}$ infection.

Conclusions Liver cancer incidence appears to have peaked among males. However, important differences in liver cancer trends by race/ethnicity and sex remain, highlighting the need for monitoring trends across different groups. Preventive interventions should focus on existing liver cancer disparities, targeting AI/ANs, females, and high-incidence groups.
\end{abstract}

Keywords Liver cancer $\cdot$ Incidence $\cdot$ Joinpoint $\cdot$ Disparities $\cdot$ Trend analysis $\cdot$ Age-period-cohort

\section{Abbreviations}

AAPC Average annual percent change

AI/AN American Indian/Alaska Native

AIC Akaike information criterion

APC Annual percent change

API Asian or Pacific Islander

ASIR Age-standardized incidence rate

HBV Hepatitis B virus

$\mathrm{HCV} \quad$ Hepatitis $\mathrm{C}$ virus

Electronic supplementary material The online version of this article (https://doi.org/10.1007/s10552-019-01237-4) contains supplementary material, which is available to authorized users.

Rafael Meza

rmeza@umich.edu

1 Department of Epidemiology, School of Public Health, University of Michigan, 1415 Washington Heights, Ann Arbor, MI 48109-2029, USA
NH Non-Hispanic

NHANES National Health and Nutrition Examination Survey

NPCR National Program of Cancer Registries

SEER Surveillance, Epidemiology, and End Results

\section{Introduction}

Liver cancer is the second most common cause of cancerrelated deaths worldwide [1]. In 2013, liver cancer had the 8th highest death rate among cancers in the US [2], with some of the fastest growth in mortality (fastest among males [2.8 average annual percent change, AAPC] and second fastest among females [2.7 AAPC], 2010-2014) and incidence (fastest among males [2.8 AAPC] and among females [3.8 AAPC], 2010-2014) [3]. Meanwhile, overall cancer incidence and mortality has been decreasing in most other 
cancer sites. Despite medical progress in the treatment of liver cancer, its 5-year survival rate remains low [4]; therefore, it is important to understand the trends of this cancer and identify high risk groups to be the focus of prevention efforts to effectively reduce this disease burden in the US.

The most significant risk factors for liver cancer are chronic Hepatitis B (HBV) or C (HCV) infection [5-9]. Globally, about $53 \%$ and $25 \%$ of liver cancers are attributable to $\mathrm{HBV}$ and $\mathrm{HCV}$, respectively [9]. However, in part because of widespread HBV vaccination programs in the US $[10,11]$ and the patterns of HCV infection among "baby boomers" (born between 1945 and 1965) and high risk populations [12-14], the attributable fraction of liver cancers due to HBV (16\%) and HCV (48\%) in the US is very different from most other regions in the world [9]. Chronic liver diseases such as cirrhosis $[6,7,9,15]$ and non-alcoholic steatohepatitis $[5,7,15,16]$ are other common precursors to liver cancer. Aflatoxin exposure [5, 6, 8, 15, 17], heavy alcohol consumption $[5,6,15,18]$, and diabetes/obesity $[5,6,15$, $18,19]$ have also been associated with the disease. Aflatoxin exposure is most commonly found in eastern Asia [17, 20], sub-Saharan Africa [17, 21-23], and Latin America [20, $24,25]$, but is not a major, direct concern in the US. However, alcohol consumption is a major public health concern [26], and the obesity/diabetes crisis is expected to worsen [27-29]. The distribution of these risk factors within the US and across the globe may help explain global differences in the trends of liver cancer.

Many studies have highlighted important racial/ethnic disparities in liver cancer with respect to incidence [30-36], mortality [31, 32, 34], survival [30, 32, 37-39], and treatment $[37,40]$. Further, sex-based disparities in incidence [31, 41-44], mortality [31, 34, 42], and survival [39] trends have also been reported. However, most incidence studies focus on short term trends or ignore important ethnic and racial groups such as American Indians/Alaska Natives (AI/ ANs), and variations by birth-cohort.

Here we update previously published studies of liver cancer incidence trends putting a focus on differences by race/ ethnicity, sex, and birth-cohort in the US using data from the Surveillance, Epidemiology and End Results (SEER) 13 registry from 1992 to 2016 and Joinpoint regression and age-period-cohort analyses.

\section{Methods}

\section{Data}

Annual delay-adjusted age-standardized liver cancer incidence rates (ASIR) from 1992 to 2016 were obtained from the SEER-13 cancer registry using the SEER*Stat software (Version 8.3.5; National Cancer Institute, US; November
2018 submission). SEER-13 rather than SEER-9 or SEER-18 was selected for our main analyses because it covers all years since new racial categories, AI/AN and Asian or Pacific Islander (API), were included in the registry. ASIRs were standardized to the 2000 US population. Incident liver cancer cases include those coded C22.0 and C22.1 according to the ICD-O-3/WHO 2008 definition. The Delay race variable was used to identify racial/ethnic groups in delay-adjusted analyses (non-Hispanic Whites, non-Hispanic Blacks, AI/ ANs, and APIs). SEER frequently only includes AI/AN cases that are in a Contract Health Service Delivery Area (CHSDA) [45] when producing statistics on AI/ANs, which is the same definition we use in our analyses.

We also created ASIRs for the following calendar year groupings: 1992-1995, 1996-2000, 2001-2005, 2006-2010, 2011-2016. ASIR ratios of racial/ethnic groups relative to Whites, males relative to females, and their corresponding confidence intervals were calculated for comparison across groups [46].

We also obtained liver cancer age-specific cases and population from SEER-13 for all race/ethnicity and sex combinations. Age-specific incidence rates for each group were calculated for single ages in each calendar year. We used the SEER non-delay-adjusted data since the delay-adjusted rates are not available for single ages.

\section{Joinpoint trend analysis}

We performed trend analyses using Joinpoint Regression Program software (Version 4.6.0.0; National Cancer Institute, US). We estimated log-linear models (log-scale for rates) of liver cancer incidence trends by race/ethnicity and sex, allowing a maximum of 4 joinpoints per model. This methodology allows for the direct calculation of the annual percent change (APC) in incidence, which is readily interpretable as well as directly comparable across different strata. The final models were selected using Bayesian information criterion (BIC) to allow for a more sensitive identification of trend change [47, 48]. AAPC was also calculated for the five most recent years (2012-2016).

Alternative analyses were performed using non-transformed incidence rates as well as the permutation test model selection method $[49,50]$. We performed additional sensitivity analyses comparing the resulting trends when using SEER-9, SEER-18, the National Program of Cancer Registries (NPCR), and the combined SEER \& NPCR data instead of SEER-13. SEER-9 allows for a comparison with a longer trend comprised of 9 of the SEER-13 registries but lacks detailed racial/ethnic data. SEER-18 allows for a comparison with a larger registry population, compromised of all the SEER-13 registries, as well as five additional registries. However, SEER-18 started in 2000, which is a shorter time-frame to analyze trends 
than SEER-13. Finally, the NPCR covers almost the entire country (96\%) [51] but lacks the continuity and quality of the SEER registries. These sensitivity analyses also included non-delay-adjusted incidence rates. The delayadjustment method adjusts incidence rates to account for delay in case reporting while non-delay-adjusted rates use raw registry data. The use of delay-adjusted rates is more conservative [52] and is often used when performing SEER analyses.

\section{Analysis of incidence by age-period-cohort}

We also performed age-period-cohort analyses of SEER-13 age-specific incidence by race/ethnicity and sex. We used the "classical" method of analysis, which fits a log-linear model with a Poisson distribution to the observed data to estimate age, period, and cohort effects [53]. To address the well-known nonidentifiability problem of age-periodcohort models, we fitted models with either cohort (ageperiod-cohort) or period (age-cohort-period) constrained to be 0 on average with 0 slope. The Akaike Information Criteria (AIC) was used to compare the relative goodness of fit of different models. All age-period-cohort analyses were done using the Epi package in the $\mathrm{R}$ statistical software ( $\mathrm{R}$ version 3.4.1). Age, period and cohort effects were modeled with natural splines, using seven degrees of freedom/ knots for age and ten for period and cohort.

We performed a sensitivity analysis estimating ageperiod-cohort trends when using SEER-9 data (1975-2016) instead of SEER-13, but restricted to all races combined, Whites (Hispanic and non-Hispanic combined) and Blacks (Hispanic and non-Hispanic combined) since SEER-9 does not allow for more detailed racial/ethnic breakdowns.

\section{Hepatitis C seroprevalence}

Finally, we provided an update to Armstrong et al. [54], where we estimated the proportion of anti-HCV-positivity (seroprevalence) in the population, which measures current or past infection, by sex both by age category and by birthcohort. We used National Health and Nutrition Examination Survey (NHANES) data from 1999 to 2002, replicating the Armstrong et al. 2006 estimates, and from 2009 to 2012. All statistical analyses were performed using R. Plots were produced using the survey and ggplot $2 \mathrm{R}$ packages. Seroprevalence estimates and corresponding $95 \%$ confidence intervals were calculated using the survey package and the logit method. A more detailed description of the data, laboratory methods used to confirm anti-HCV-positivity, statistical analysis, and interpretation can be found in Online Appendix G.

\section{Results}

\section{Age-standarized incidence rate analyses}

Table 1 describes ASIRs, number of cases, and total base populations by race/ethnicity and sex from 1992 to 2016 . Whites had the lowest incidence rate among all groups over the period of analysis.

Figure 1 shows the corresponding incidence rates and joinpoint trend lines by race/ethnicity, sex and year. The Figure shows that incidence trends in liver cancer have increased for all combinations of race/ethnicity and sex, but that for males the rates might have peaked. Until around 2010, APIs had been the most affected racial/ethnic group. However, among both males and females, AI/ANs (and, to a lesser extent, Hispanics) have shown consistent increases in incidence recently surpassing APIs.

Table 1 Age-standardized delay-adjusted incidence rate (per 100,000) of liver cancer and number of cases by race/ethnicity and sex, 1992-2016

\begin{tabular}{lrrl}
\hline & $1992-2016$ & & \\
\cline { 2 - 4 } & Rate & Count & Population \\
\hline Both & & & \\
All & 7.5 & 75,276 & $996,176,800$ \\
AI/AN & 13.6 & 867 & $10,456,661$ \\
White & 5.3 & 34,816 & $553,152,154$ \\
Black & 9.7 & 8,537 & $107,341,373$ \\
API & 14.3 & 15,833 & $121,173,133$ \\
Hispanic & 11.8 & 13,072 & $208,116,380$ \\
Males & & & \\
All & 11.5 & 51,293 & $491,577,567$ \\
AI/AN & 18.6 & 553 & $5,164,609$ \\
White & 8.1 & 24,382 & $273,448,023$ \\
Black & 15.8 & 6,149 & $50,928,700$ \\
API & 21.8 & 10,898 & $58,344,975$ \\
Hispanic & 17.9 & 9,181 & $105,754,350$ \\
Females & & & \\
All & 4.1 & 21,983 & $504,599,233$ \\
AI/AN & 9.5 & 314 & $5,292,052$ \\
White & 2.9 & 10,434 & $279,704,131$ \\
Black & 4.9 & 2,388 & $56,412,673$ \\
API & 8.2 & 4,935 & $62,828,158$ \\
Hispanic & 6.7 & 3,891 & $102,362,030$ \\
\hline & & & \\
\hline
\end{tabular}

Rates are per 100,000 and are age-standardized to the 2000 US standard population; White and Black exclude those who identify as Hispanic; All includes all races combined and both those who identify as Hispanic and Non-Hispanic; Hispanic represents those from any race who identify as Hispanic

Case counts from all racial groups do not add up to total cases due to cases in database with unknown race/ethnicity. Cases with unknown race/ethnicity do not effect population counts

AI/AN, American Indian/Alaska Native; API, Asian or Pacific Islander 

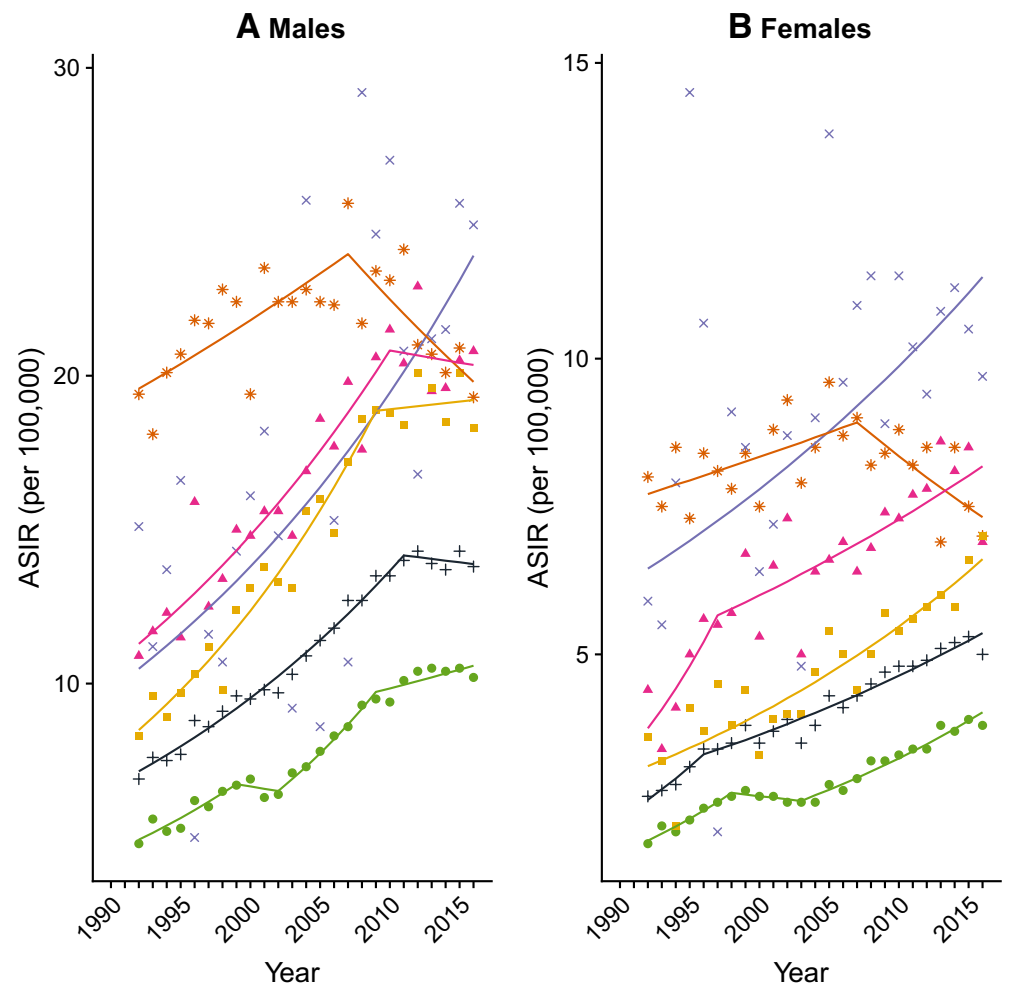

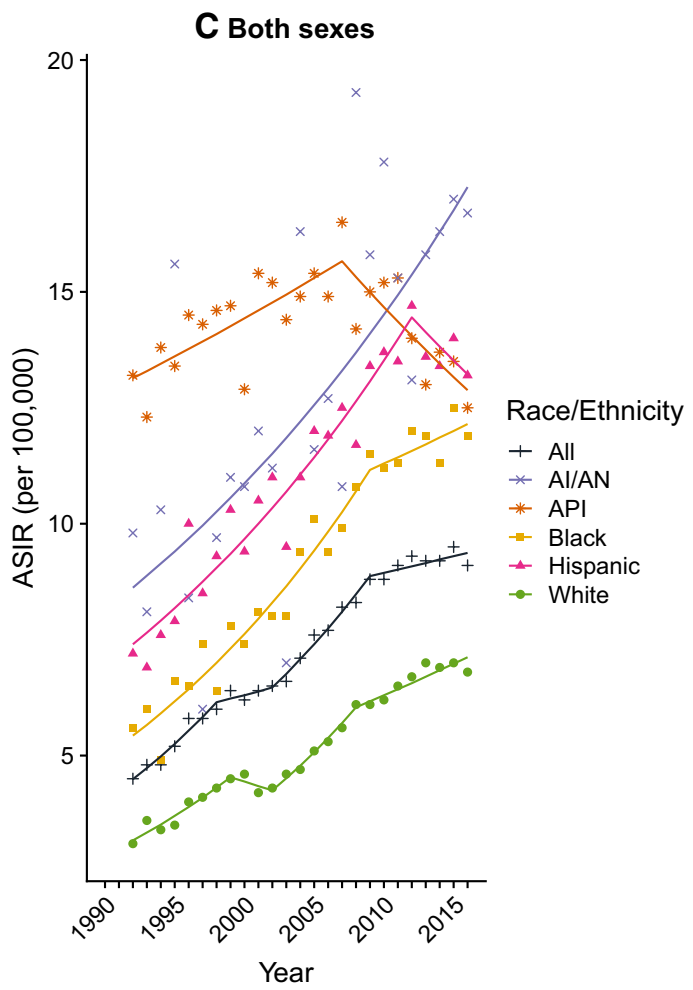

Fig. 1 Delay-adjusted age-standardized liver cancer incidence rates (ASIR) by race/ethnicity for males and females. Rates are age standardized to the 2000 US population. White and Black exclude those who identify as Hispanic; All includes all races combined and both those who identify as Hispanic and non-Hispanic; Hispanic repre- sents those from any race who identify as Hispanic. Lines represent the joinpoint models prediction, and points represent observed data in SEER-13 registry (AI/AN, American Indian and Alaska Native; API, Asian or Pacific Islander)

incidence than Whites throughout the period of analysis. However, APIs had a fourfold higher incidence than Whites in 1992-1995, but their relative incidence decreased to a twofold higher level in 2011-2016. As shown in Fig. 2b, for most racial/ethnic groups, males had about a threefold higher incidence than females, except among AI/ANs, which had about a twofold male-to-female ratio.

Alternative trend analyses in the non-transformed incidence scale, non-delay-adjusted rates, or with different model selection criteria are referenced in the discussion section below, and figures and tables of the results are shown in the Appendices. In general, the results for these sensitivity analyses are consistent with our conclusions, with only slight deviations.

\section{Age-period-cohort analyses}

For all race/ethnicity and sex groups except Hispanic females, age-cohort models fit the data better than ageperiod models, suggesting that cohort correlates better than year of diagnosis (period) with liver cancer incidence trends (model AICs are shown in Online Appendix F). The top panels in Fig. 3 show the estimated cohort effects, relative 
Table 2 Log-transformed joinpoint trends in delay-adjusted liver cancer incidence rates by race/ethnicity and sex

\begin{tabular}{|c|c|c|c|c|c|c|c|c|c|c|}
\hline \multirow[t]{2}{*}{ Sex } & \multirow[t]{2}{*}{ Race } & \multicolumn{2}{|l|}{ Trend 1} & \multicolumn{2}{|l|}{ Trend 2} & \multicolumn{2}{|l|}{ Trend 3} & \multicolumn{2}{|l|}{ Trend 4} & \multirow[b]{2}{*}{$\begin{array}{l}2012- \\
2016 \text { AAPC }\end{array}$} \\
\hline & & Years & APC & Years & APC & Years & APC & Years & APC & \\
\hline \multirow[t]{6}{*}{ Both } & All & 1992-1998 & $5.39 *$ & 1998-2002 & 1.27 & 2002-2009 & $4.62 *$ & 2009-2016 & 0.80 & 0.8 \\
\hline & $\mathrm{AI} / \mathrm{AN}$ & 1992-2016 & $2.93^{*}$ & & & & & & & $2.9^{*}$ \\
\hline & White & 1992-1999 & $5.23 *$ & 1999-2002 & -2.08 & 2002-2008 & $6.03 *$ & 2008-2016 & $2.07 *$ & $2.1^{*}$ \\
\hline & Black & 1992-2009 & $4.33^{*}$ & 2009-2016 & 1.22 & & & & & 1.2 \\
\hline & API & 1992-2007 & $1.18 *$ & 2007-2016 & $-2.15^{*}$ & & & & & $-2.2^{*}$ \\
\hline & Hispanic & 1992-2012 & $3.40^{*}$ & 2012-2016 & -2.21 & & & & & -2.2 \\
\hline \multirow[t]{6}{*}{ Males } & All & 1992-2011 & $3.67 *$ & 2011-2016 & -0.39 & & & & & -0.4 \\
\hline & $\mathrm{AI} / \mathrm{AN}$ & 1992-2016 & $3.49 *$ & & & & & & & $3.5^{*}$ \\
\hline & White & 1992-1999 & $4.54^{*}$ & 1999-2002 & -1.08 & 2002-2009 & $5.90 *$ & 2009-2016 & 1.21 & 1.2 \\
\hline & Black & 1992-2009 & $4.80^{*}$ & 2009-2016 & 0.27 & & & & & 0.3 \\
\hline & API & 1992-2007 & $1.35^{*}$ & 2007-2016 & $-2.09 *$ & & & & & $-2.1^{*}$ \\
\hline & Hispanic & 1992-2010 & $3.46^{*}$ & 2010-2016 & -0.38 & & & & & -0.4 \\
\hline \multirow[t]{6}{*}{ Females } & All & 1992-1996 & $6.76^{*}$ & 1996-2016 & $2.45 *$ & & & & & $2.4^{*}$ \\
\hline & AI/AN & 1992-2016 & $2.39 *$ & & & & & & & $2.4^{*}$ \\
\hline & White & 1992-1998 & $6.23 *$ & 1998-2003 & -1.05 & 2003-2016 & $3.65 *$ & & & $3.7 *$ \\
\hline & Black & 1992-2016 & $3.19 *$ & & & & & & & $3.2^{*}$ \\
\hline & API & 1992-2007 & $0.97^{*}$ & 2007-2016 & $-2.18 *$ & & & & & $-2.2^{*}$ \\
\hline & Hispanic & 1992-1997 & $8.56^{*}$ & 1997-2016 & $1.96^{*}$ & & & & & $2.0^{*}$ \\
\hline
\end{tabular}

White and Black exclude those who identify as Hispanic; All includes all races combined and both those who identify as Hispanic and NonHispanic; Hispanic represents those from any race who identify as Hispanic

$A A P C$, Average annual percent change from 2012 to 2016; APC, Annual percent change; AI/AN, American Indian and Alaska Native; API, Asian or Pacific Islander

*Significantly different from zero, $p<0.05$

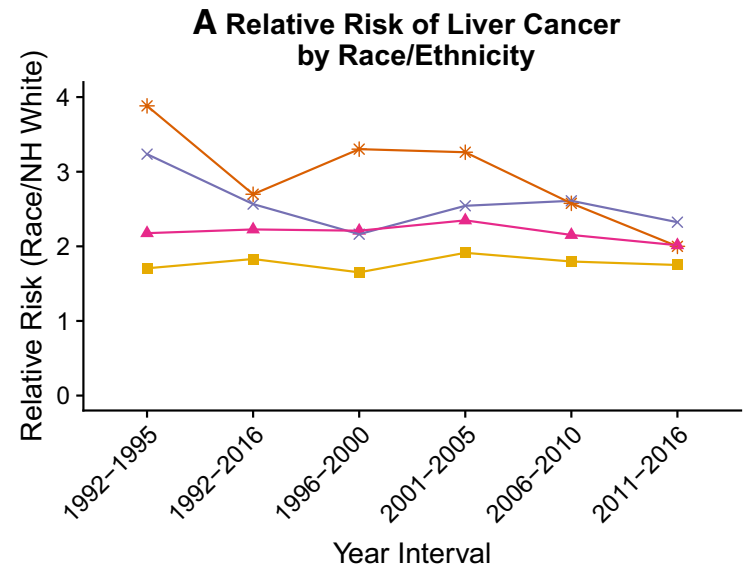

Fig. 2 Relative ratio of liver cancer incidence for racial/ethnic group with respect to non-Hispanic Whites (a), and for males with respect to females (b). White and Black exclude those who identify as Hispanic; All includes all races combined and both those who identify

to the 1930 birth-cohort, from age-period-cohort models with the period effects constrained to be 0 on average with 0 slope (age-cohort-period model). The figure shows the striking cohort trends of liver cancer incidence, particularly for men, with a sharp increase in relative incidence beginning

\section{B Relative Risk of Liver Cancer by Sex by Race/Ethnicity}

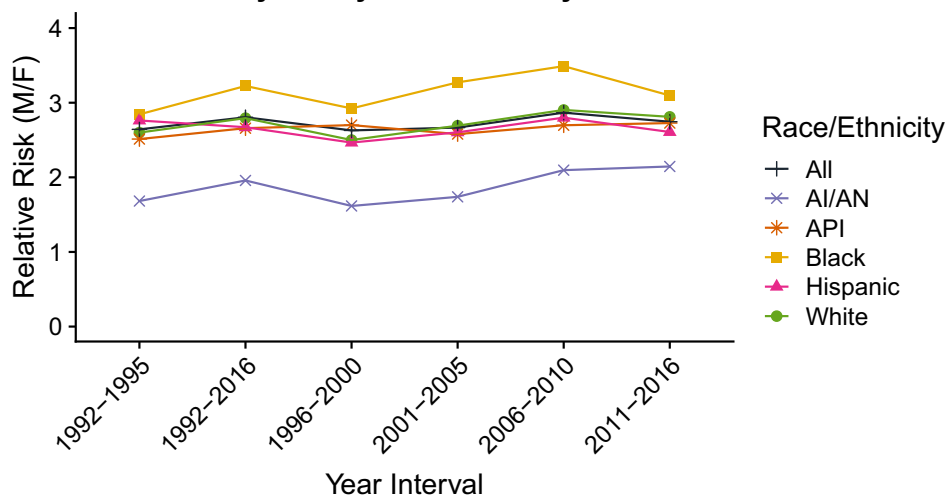

as Hispanic and non-Hispanic; Hispanic represents those from any race who identify as Hispanic (AI/AN, American Indian and Alaska Native; API, Asian or Pacific Islander)

with the 1940s birth-cohorts and around the 1950-1955 cohorts. This pattern is particularly noticeable for Blacks, but also seen in all other groups except for APIs. Notably, the male liver cancer incidence has decreased steadily ever since, except in AI/ANs, which continue to have increasing 
Fig. 3 Estimated cohort and age-effects from age-periodcohort models of liver cancer incidence by race/ethnicity with period effects constrained to be 0 on average with 0 slope (age-cohort-period model). Top panels show the cohort effects, which represent the liver cancer incidence relative to that of the 1930 birth-cohort, by race/ethnicity and sex. Bottom panels show the estimated age-specific incidence rates by race/ethnicity and sex for the 1930 birthcohort (age-effects). White and Black exclude those who identify as Hispanic; All includes all races combined and both those who identify as Hispanic and non-Hispanic; Hispanic represents those from any race who identify as Hispanic (AI/AN, American Indian and Alaska Native; API, Asian or Pacific Islander)
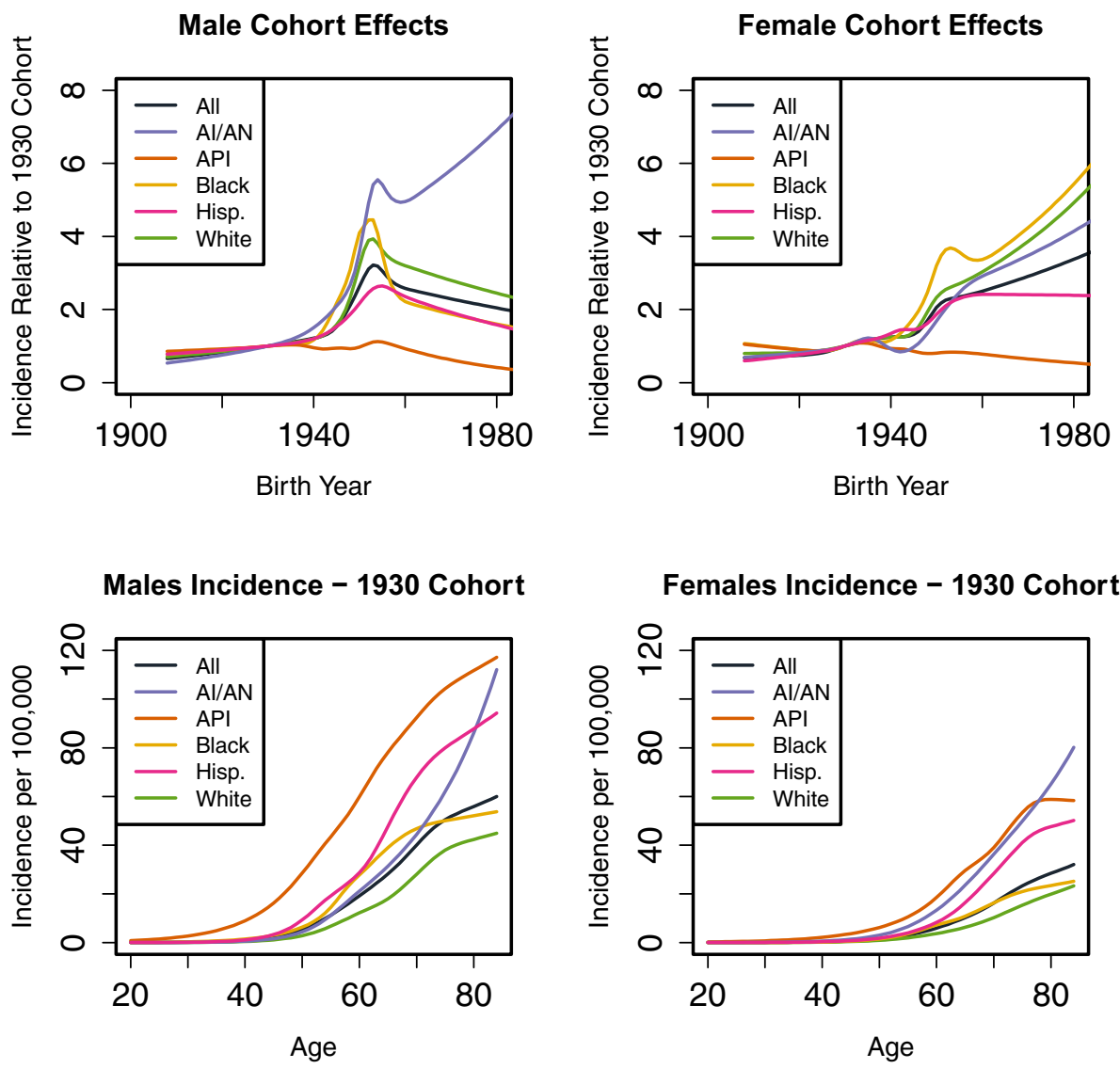

incidence by cohort. In contrast, the incidence continues to increase by cohort for all female groups except APIs. The corresponding estimated age-specific incidence rates by race/ethnicity and sex for the 1930 birth-cohort (age-effects) are shown in the bottom panels.

Alternative age-period-cohort analyses using SEER-9 data (1975-2016) for all races combined, Whites (Hispanics and non-Hispanics combined) and Blacks (Hispanics and non-Hispanics combined) are shown in Online Appendix $\mathrm{H}$. These show consistent patterns of liver cancer incidence by birth-cohort, sex and race as when using SEER-13, with age-cohort models also fitting the data better than age-period models.

Figure 4 shows the proportion of anti-HCV-positivity (seroprevalence) using NHANES data by sex by age category (Fig. 4a, b) and by birth-cohort (Fig. 4c, d). Figure 4a, b show peaks among 40-49 year olds for both males and females according to 1999-2002 NHANES data and peaks among 50-59 year olds for both males and females according to 2009-2012 NHANES data. The age category estimates for the 2009-2012 data are similar to the same estimate in the younger 10-year age category for the 1999-2002 data. Figure $4 \mathrm{c}$ shows a peak among males born in the 1955-1959 birth-cohort with a steep decline in anti-HCV-positivity in younger cohorts in both the 1999-2002 and 2009-2012
NHANES data. Figure 4d shows relatively low and consistent anti-HCV-positivity estimates among females with a drop off in younger birth-cohorts in both the 1999-2002 and 2009-2012 NHANES data.

\section{Discussion}

We analyzed trends in liver cancer incidence in the US by race/ethnicity and sex from 1992 to 2016 . Our study shows that overall liver cancer incidence has peaked in males, while it continues to increase in females, but at a lower rate than in the 1990s. This is consistent across most racial/ethnic groups, with the exception of AI/ANs and APIs. The incidence rates among AI/ANs are increasing considerably in both males and females and have overtaken APIs as the racial/ethnic group facing the highest burden of liver cancer incidence. In contrast, incidence among APIs has been decreasing considerably in both sexes since the mid-to-late 2000s. These trends largely reflect changes in liver cancer by birth-cohort, which dramatically increased for those born around the 1950s, and seem consistent with cohort patterns of Hepatitis $\mathrm{C}$ infection. Trends in younger male cohorts have decreased; however, this decrease is not seen in females nor in AI/ANs. 

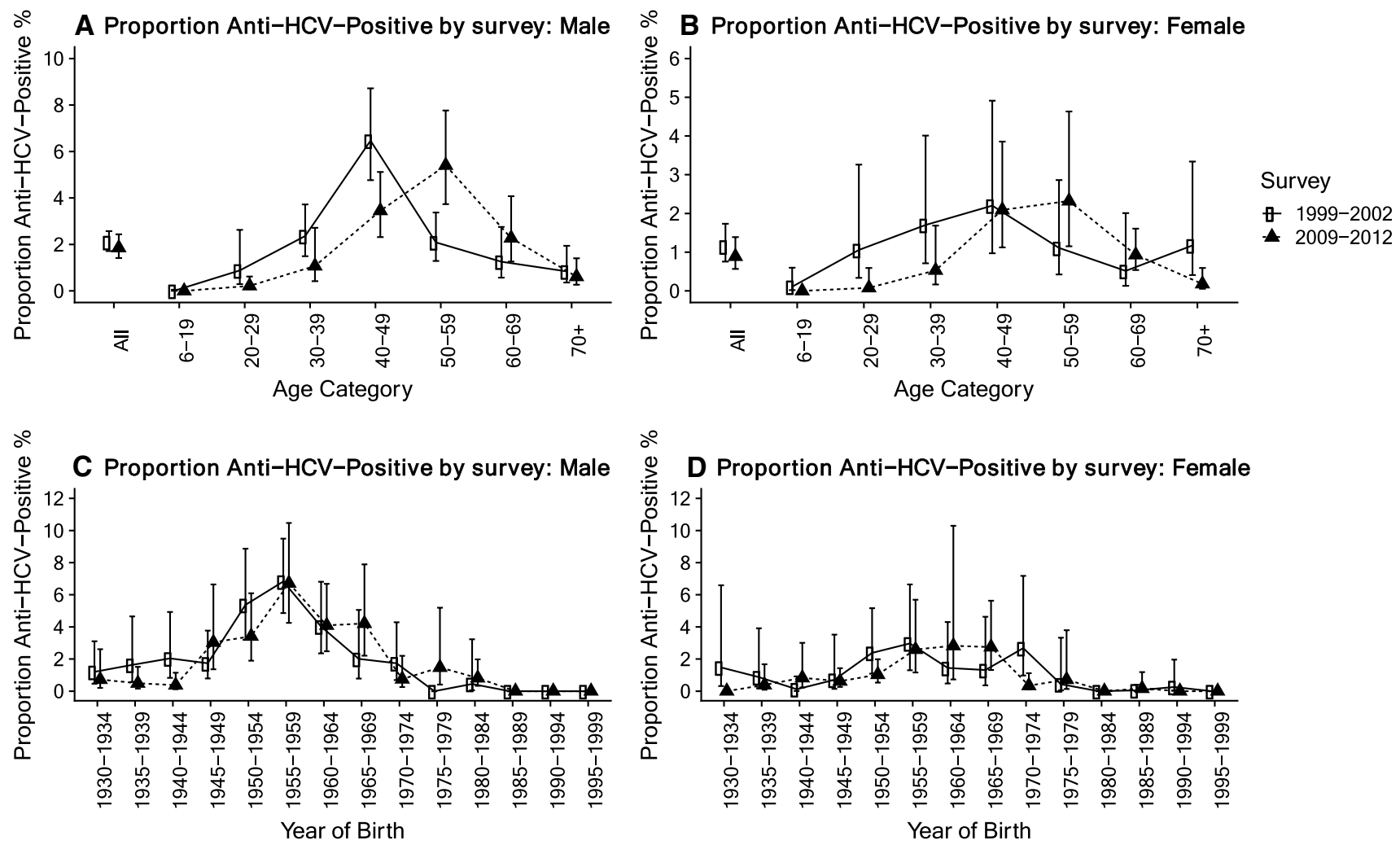

Fig. 4 Estimated proportion of anti-HCV-positivity in US population using NHANES data from 1999-2002 and 2009-2012. Panels $\mathrm{A}$ and $\mathrm{B}$ show the estimated proportion of anti-HCV-positivity and corresponding $95 \%$ confidence intervals by age group for males and

females, respectively. Panels C and D show the estimated proportion of anti-HCV-positivity and corresponding $95 \%$ confidence intervals by birth-cohort for males and females, respectively

Our results show that AI/ANs are becoming the group most disproportionately affected by liver cancer and thus merit tailored prevention efforts. Moreover, the maleto-female relative risk for liver cancer incidence in $\mathrm{AI} /$ ANs was lower than those in other racial/ethnic groups, indicating that the sex difference in liver cancer incidence among AI/ANs is less prominent than in other racial/ethnic groups. In a recent incidence analysis from 1999 to 2009 using Indian Health Service linked national cancer registry data, Melkonian et al. [36] also found that liver cancer incidence is increasing among AI/ANs. In addition, they found that alcohol use and obesity were correlated with liver cancer incidence among AI/ANs by region and that AI/ANs were diagnosed at later stages than whites. Prevention efforts for AI/ANs should then include strategies to reduce alcohol use and obesity rates and to increase surveillance for early detection of liver cancer and its related conditions, in addition to ongoing HBV vaccination and $\mathrm{HCV}$ infection prevention programs.

The observed trends among APIs are in line with the observed decrease in liver cancer incidence in Asia [17]. APIs included in the data are a combination of US-born and foreign-born persons. Thus, the trends within the API

population could be explained in part by changes in risk factor exposure (e.g., reduction of aflatoxin exposures [17]) and the introduction of the Hepatitis B vaccine in Asia. However, we were unable to explore differences in liver cancer incidence by nationality due to lack of country of origin information in SEER data.

The identified cohort patterns of liver cancer are consistent with the dramatic increase in Hepatitis C infections [54, 55] and other liver conditions [56] among baby boomers, particularly males. In particular, using NHANES data from 1999 to 2002, Armstrong et al. reported the higher burden of Hepatitis C among those born between 1945 and 1964, with a higher seroprevalence in males, and non-Hispanic Blacks. Figure 4 shows updated estimates including NHANES data from 1999 to 2002 and 2009 to 2012, demonstrating that this pattern remains until today. The dramatic increase of Hepatitis $\mathrm{C}$ rates among baby boomers has led the Centers for Disease Control and Prevention to establish specific screening recommendation for this population group [55]. The strong cohort patterns in liver cancer incidence (Fig. 3), which match closely the cohort patterns of Hepatitis $\mathrm{C}$ seroprevalence (Fig. 4), suggest that current liver cancer rates are influenced greatly by the patterns in Hepatitis $\mathrm{C}$ infections. 
These observed cohort patterns provide additional support to our findings of a decreasing liver cancer trend in males, which is now coming back to its baseline trend as those historically affected by Hepatitis $\mathrm{C}$ become a smaller fraction of the population. However, this decrease is not seen in females, who have in general lower liver cancer rates, which suggests that other factors might be in play behind the increase of liver cancer by cohort in females.

Limitations of the study include the following: First, the ecologic nature of the study does not allow for causal inferences. The SEER registry does not contain information about Hepatitis B and C infections, Hepatitis B vaccination history, chronic liver conditions, drinking behavior, smoking and other relevant risk factors for liver cancer. However, the registry maintains high-quality data and continuity in structure and variable definitions across time, which is paramount for drawing valid conclusions about incidence rates trends over longer periods of time. Additionally, by choosing SEER-13, we limited our period of analysis to 1992-2016 and excluded the population from the five cancer registries that were added in 2000 to SEER-18. Nonetheless, we performed sensitivity analyses to examine trends of liver cancer incidence rates using different versions of the SEER registry covering a longer period (SEER-9) or a larger population (SEER-18), and with national data from the NPCR. Delay-adjusted rates were not available for NPCR or for combined SEER and NPCR data. The sensitivity analyses show that our conclusions on liver cancer incidence trends by sex and race/ethnicity are generally robust regardless of the dataset choice, but with some differences in the level of ASIR (Online Appendix E). In particular, it appears that the SEER-13 incidence rates are generally higher, reflecting potential high liver cancer incidence in the population that was added to the SEER-9 registries: Los Angeles, San JoseMonterey, Rural Georgia, and Alaska Natives. These sites were selected to oversample minority populations that have a higher burden of liver cancer, which allowed us to perform stratified analyses by race/ethnicity and sex. Similarly, ageperiod-cohort sensitivity analyses using the SEER-9 data show that the cohort patterns found in the main analyses with SEER-13 hold when looking at incidence data going back to the mid 1970s, despite not being able to stratify these analyses by detailed racial/ethnic groups as with SEER-13.

Our study also has many strengths. The study benefits from the high-quality, comparable data collected by the SEER registry. Further, joinpoint regression is an objective approach to characterize trends in incidence data employing validated statistical methods [57-60]. This analysis used the BIC model selection method, which is more sensitive at detecting trends than the permutation test, the default model selection method in the Joinpoint Regression Program [61, 62]. We selected the BIC criteria to more aggressively detect statistically significant changes in trends, which revealed more clearly recent shifts in trajectory. Sensitivity analyses using the less sensitive permutation test showed that our conclusions are generally robust to the choice of method, and consistent with the cohort patterns in incidence. Moreover, our analyses of incidence through 2016 update previous analyses based on SEER data [3, 31, 32, 39, 63] and show more decisively that the trends have changed considerably in recent years, having reached a peak overall and in males but not in females, with variations by race/ethnicity. In addition, the age-period-cohort analyses complement and enhance the joinpoint analyses, since these focus on crude rates and capture incidence variations by age and other temporal factors, such as cohort and period. Finally, by using SEER-13 we were able to examine in detail the rates of liver cancer incidence among AI/ANs, a group who tend to be overlooked and understudied in cancer and health research [64]. Previous analyses using SEER data have intentionally excluded $\mathrm{AI} / \mathrm{ANs}$ because of their small sample size or case counts $[31,65,66]$.

Past studies have described trends of liver cancer in different groups and periods, although haven't focused on cohort trends. Of note, Altekruse et al. [31] examined trends in liver cancer incidence by age, race/ethnicity, and sex and mortality by age, geography, race/ethnicity, and sex from 2000 to 2010 using SEER-18 data. While this study benefited from an increased coverage area due to the inclusion of 5 additional registries, the length of time over which trends were observed was shorter. Additionally, their analysis did not include AI/ANs, allowed for only two trends (one joinpoint), and did not find the decreasing trends observed in recent years. Our conclusions regarding racial/ethnic trends between 2000 and 2010 are in line with Altekruse et al.'s, while our analyses suggest the magnitude of increase is smaller. Notably, however, we conclude that the incidence rate among Blacks has leveled off while Altekruse et al. found that it was still increasing. Regardless of whether the incidence is still increasing or has peaked, the incidence rates among Blacks are relatively high and thus deserve attention. Moreover, our analysis reports trends by race/ethnicity and sex, and characterizes incidence patterns by birth-cohort, thus complementing the previous findings by Altekruse et al.

Njei et al. [39] used SEER-9 to examine trends in liver cancer incidence and mortality by sex and by stage from 1973 to 2010. Their period of analysis was longer than ours and a sensitivity analysis was performed using SEER-18 from 2000 to 2011. The authors concluded that there is a non-significant increase in the overall incidence rate of liver cancer for the first time in four decades but did not analyze trends by race/ ethnicity. Njei et al. also anticipated that the peak of liver cancer incidence would occur in 2017. Our analysis suggests that the overall peak may have already occurred, particularly for males, driven by the decrease in incidence by cohort 
for people born after the 1950s. Of note, our findings disagree with their conclusions about current incidence trends by sex. Njei et al. concluded that the incidence in males is still increasing while we found that the increase has stopped. Among females, they concluded that the rate is decreasing while it has actually been increasing since 1992, although the increase slowed down in the late 1990s.

In their 2017 paper, Islami et al. [32] reported liver cancer incidence and survival rates and trends by race/ethnicity and mortality by race/ethnicity and state. They analyzed mortality trends using joinpoint regression and found that while mortality is still increasing for some groups, the increase appears to be slowing down in recent years (non-statistically significant APC), which is consistent with our findings. Islami et al. also examined liver cancer incidence but did not perform a joinpoint regression analysis and used data only up to 2013.

Finally, two more recent publications also report liver cancer incidence trends by race/ethnicity and sex. Cronin et al. [3] reports significant AAPCs from 2010 to 2014 of 2.8 and 3.8 for males and females respectively using a combination of SEER and NPCR delay-adjusted incidence data. A more recent report, Ward et al. [67] finds significant AAPCs from 2011 to 2015 of 2.7 and 3.8 for males and females respectively. However, when replicating our joinpoint analyses using combined non-delay-adjusted SEER and NPCR data from 2001 to 2016, we find trends consistent with our main findings (Online Fig. I1). This shows that the apparent differences between our results and those in Cronin et al. and Ward et al. stem from the use of different metrics/approaches (AAPC vs joinpoint trend analysis). Interestingly, when comparing the AAPCs in Cronin et al. and Ward et al., several show a decrease in liver cancer incidence AAPCs in the more recent period (2011-2015), e.g., (from 2.6* to $2.1^{*}$ in Black men) suggesting that incidence has indeed peaked. Also consistent with our findings, Siegel et al. [68], using SEER-9 data from 1975 to 2015, found that incidence rates have stopped increasing in recent years among males (nonsignificant 1.0 AAPC from 2011 to 2015). Our main and sensitivity analyses show that this is the case in SEER-13, SEER-9, SEER-18 and the combined SEER and NPCR data when including data through 2016.

Several reasons could explain the plateau in liver cancer incidence rates in males in recent years. First, recent declines in the prevalence of $\mathrm{HCV}$ infection (liver cancer OR 8.2, 95\% CI 6.7-9.9) [69] have been reported [70]. As suggested above, the strong similarity of the cohort patterns of liver cancer and Hepatitis $\mathrm{C}$ seroprevalence suggest that the latter might be partly responsible for the liver cancer trends in recent decades, particularly in males, as more recent birth-cohorts with lower exposures to HCV become a larger proportion of the population. Second, we could finally be observing the impact of the Hepatitis B vaccination program, which started in the 1980 s, resulting in decreases of HBV prevalence [71, 72]. This suggests that the burden of liver cancer attributable to HBV (OR 15.5, 95\% CI 13.6-17.8) [69] may have also reached its peak in the US. Third, we could also be observing a delayed impact of a decrease in alcohol consumption on liver cancer incidence $(\mathrm{RR}=1.08,95 \%$ CI 1.04-1.11; for one alcoholic drink per day) [73]. Greenfield and Kerr [74] documented that ethanol consumption reached a peak in the US in the early 1980s, which could be now reflected in liver cancer; however, recent data suggest that alcohol consumption began increasing again in 1998 [75]. The decreases in smoking in the past decades might also be contributing [76], although the association between smoking and liver cancer is comparatively modest (OR 1.51, 95\% CI 1.37-1.67) [77]. Finally, sex and racial/ethnic variations in the prevalence of these risk factors could explain the observed differences we found in liver cancer trends.

In summary, our analyses suggest for the first time that liver cancer incidence in males might be reaching its peak. In particular, we found that incidence rates have stopped increasing in males for most racial/ethnic groups. But these rates continue to increase in females, although at a lower rate than in the 1990s. We also found that liver cancer incidence rates in $\mathrm{AI} / \mathrm{ANs}$ continue to increase and have overtaken all other racial/ethnic groups, and that rates among APIs have decreased considerably since the mid-tolate 2000s. These age-adjusted trends are largely explained by patterns of liver cancer incidence by birth-cohort. Future studies should explore liver cancer disparities in greater detail, with an emphasis on AI/ANs, females, and other high-incidence groups. Future research should also look to understand what factors are behind the changes and differences in racial/ethnic and sex-based incidence rate trends, and preventive interventions should continue to focus on reducing exposure to the most relevant liver cancer risk factors, such as $\mathrm{HBV}$ or $\mathrm{HCV}$ infection, obesity, alcohol, and smoking.

Acknowledgments Research reported in this publication was partially supported by the National Cancer Institutes of Health under Award Number P30CA046592. The content is solely the responsibility of the authors and does not necessarily represent the official views of the National Institutes of Health.

\section{Compliance with ethical standards}

Conflict of interest The authors declare no conflict of interest.

Open Access This article is distributed under the terms of the Creative Commons Attribution 4.0 International License (http://creativeco mmons.org/licenses/by/4.0/), which permits unrestricted use, distribution, and reproduction in any medium, provided you give appropriate credit to the original author(s) and the source, provide a link to the Creative Commons license, and indicate if changes were made. 


\section{References}

1. International Agency for Research on Cancer (2012) GLOBOCAN 2012: Cancer Incidence, Mortality and Prevalence Worldwide. https://globocan.iarc.fr/old/burden.asp?selec tion_pop $=207840 \&$ Text-p $=$ United + States + of + America \&selec tion_cancer $=14070 \&$ Text-c $=$ Liver $\& p Y e a r=18 \&$ type $=0 \&$ windo $\mathrm{w}=1 \&$ submit $=$ Execute. Accessed 27 Nov 2016.

2. Centers for Disease Control and Prevention (2013) United States Cancer statistics: 2013 top ten cancers. https://nccd.cdc.gov/uscs/ toptencancers.aspx. Accessed 31 Mar 2017.

3. Cronin KA, Lake AJ, Scott S et al (2018) Annual report to the nation on the status of cancer, part I: National cancer statistics. Cancer 124(13):2785-2800. https://doi.org/10.1002/cncr.31551

4. National Cancer Institute. Cancer trends progress report. https:// progressreport.cancer.gov/diagnosis/incidence. Accessed 31 Mar 2017.

5. McGlynn KA, London WT (2011) The global epidemiology of hepatocellular carcinoma: present and future. Clin Liver Dis 15(2):223-243. https://doi.org/10.1016/j.cld.2011.03.006

6. El-Serag HB (2012) Epidemiology of viral hepatitis and hepatocellular carcinoma. Gastroenterology 142(6):1264-1273. https:// doi.org/10.1053/j.gastro.2011.12.061

7. Kew MC (2010) Epidemiology of chronic hepatitis B virus infection, hepatocellular carcinoma, and hepatitis B virus-induced hepatocellular carcinoma. Pathol Biol 58(4):273-277. https:// doi.org/10.1016/j.patbio.2010.01.005

8. Liang X, Bi S, Yang W et al (2009) Epidemiological serosurvey of Hepatitis B in China-declining HBV prevalence due to hepatitis B vaccination. Vaccine 27(47):6550-6557. https://doi. org/10.1016/j.vaccine.2009.08.048

9. Perz JF, Armstrong GL, Farrington LA, Hutin YJF, Bell BP (2006) The contributions of hepatitis $B$ virus and hepatitis $C$ virus infections to cirrhosis and primary liver cancer worldwide. J Hepatol 45(4):529-538. https://doi.org/10.1016/j.jhep.2006.05.013

10. Ropero Álvarez AM, Pérez-Vilar S, Pacis-Tirso C et al (2017) Progress in vaccination towards hepatitis $\mathrm{B}$ control and elimination in the Region of the Americas. BMC Public Health 17(1):325. https ://doi.org/10.1186/s12889-017-4227-6

11. Lu P, Byrd KK, Murphy TV (2009) Weinbaum C (2011) Hepatitis B vaccination coverage among high-risk adults $18-49$ years, US. Vaccine. 29(40):7049-7057. https://doi.org/10.1016/j.vacci ne.2011.07.030

12. Jemal A, Fedewa SA (2017) Recent hepatitis C virus testing patterns among baby boomers. Am J Prev Med 53(1):e31-e33. https ://doi.org/10.1016/j.amepre.2017.01.033

13. Jemal A, Fedewa SA (2015) Prevalence of hepatitis C Virus testing in cohorts born between 1945 and 1965 in the US. Am J Prev Med 48(5):e7-e9. https://doi.org/10.1016/j.amepre.2014.12.002

14. Edlin BR, Eckhardt BJ, Shu MA, Holmberg SD, Swan T (2015) Toward a more accurate estimate of the prevalence of hepatitis $C$ in the United States. Hepatology 62(5):1353-1363. https://doi. org/10.1002/hep. 27978

15. Hamed MA, Ali SA (2013) Non-viral factors contributing to hepatocellular carcinoma. World J Hepatol 5(6):311-322. https://doi. org/10.4254/wjh.v5.i6.311

16. Neuschwander-Tetri BA, Caldwell SH (2003) Nonalcoholic steatohepatitis: summary of an AASLD single topic conference. Hepatology 37(5):1202-1219. https://doi.org/10.1053/jhep.2003.50193

17. Chen J-G, Egner PA, Ng D et al (2013) Reduced aflatoxin exposure presages decline in liver cancer mortality in an endemic region of China. Cancer Prev Res 6(10):1038-1045. https://doi. org/10.1158/1940-6207.CAPR-13-0168

18. Loomba R, Yang HI, Su J et al (2013) Synergism between obesity and alcohol in increasing the risk of hepatocellular carcinoma: a prospective cohort study. Am J Epidemiol 177(4):333-342. https://doi.org/10.1093/aje/kws252

19. Calle EE, Rodriguez C, Walker-Thurmond K, Thun MJ (2003) Overweight, obesity, and mortality from cancer in a prospectively studied cohort of US adults. N Engl J Med. 348(17):1625-1638. https://doi.org/10.1056/NEJMoa021423

20. Wu F, Guclu H (2012) Aflatoxin regulations in a network of global maize trade. PLoS ONE 7(9):e45151. https://doi. org/10.1371/journal.pone.0045151

21. Turner P, Sylla A, Gong Y et al (2005) Reduction in exposure to carcinogenic aflatoxins by postharvest intervention measures in west Africa: a community-based intervention study. Lancet 365(9475):1950-1956. https://doi.org/10.1016/S0140 -6736(05)66661-5

22. Probst C, Bandyopadhyay R, Cotty PJ (2014) Diversity of aflatoxin-producing fungi and their impact on food safety in subSaharan Africa. Int J Food Microbiol 174:113-122. https://doi. org/10.1016/j.ijfoodmicro.2013.12.010

23. Hell K, Mutegi C (2011) Aflatoxin control and prevention strategies in key crops of Sub-Saharan Africa. Afr J Microbiol Res 5(5):459-466

24. Resnik S, Lourdes Costarrica M, Pacin A (1995) Mycotoxins in Latin America and the Caribbean. Food Control 6(1):19-28

25. Torres O, Matute J, Gelineau-van Waes J et al (2015) Human health implications from co-exposure to aflatoxins and fumonisins in maize-based foods in Latin America: guatemala as a case study. World Mycotoxin J 8(2):143-159. https://doi. org/10.3920/WMJ2014.1736

26. National Institute on Alcohol Abuse and Alcoholism. Alcohol Facts and Statistics.

27. Wang Y, Beydoun MA, Liang L, Caballero B, Kumanyika SK (2008) Will all Americans become overweight or obese? Estimating the progression and cost of the us obesity epidemic. Obesity 16(10):2323-2330. https://doi.org/10.1038/ oby. 2008.351

28. Wang Y, Beydoun MA (2007) The obesity epidemic in the united states gender, age, socioeconomic, racial/ethnic, and geographic characteristics: a systematic review and meta-regression analysis. Epidemiol Rev 29(1):6-28. https://doi.org/10.1093/epirev/mxm00 7

29. Mokdad AH (2001) The continuing epidemics of obesity and diabetes in the United States. JAMA 286(10):1195. https://doi. org/10.1001/jama.286.10.1195

30. Ward E, Jemal A, Cokkinides V et al (2004) Cancer disparities by race/ethnicity and socioeconomic status. Cancer J Clin 54:78-93

31. Altekruse SF, Henley SJ, Cucinelli JE, McGlynn KA (2014) Changing hepatocellular carcinoma incidence and liver cancer mortality rates in the United States. Am J Gastroenterol 109(4):542-553. https://doi.org/10.1038/ajg.2014.11

32. Islami F, Miller KD, Siegel RL, Fedewa SA, Ward EM, Jemal A (2017) Disparities in liver cancer occurrence in the United States by race/ethnicity and state. CA Cancer J Clin 67(4):273-289. https ://doi.org/10.3322/caac.21402

33. Pham C, Fong T-L, Zhang J, Liu L (2018) Striking racial/ethnic disparities in liver cancer incidence rates and temporal trends in California, 1988-2012. J Natl Cancer Inst 110:1256-1269. https ://doi.org/10.1093/jnci/djy051

34. White MC, Espey DK, Swan J, Wiggins CL, Eheman C, Kaur JS (2014) Disparities in cancer mortality and incidence among American Indians and Alaska natives in the United States. Am J Public Health 104(SUPPL. 3):377-387. https://doi.org/10.2105/ AJPH.2013.301673

35. Yang B, Liu JB, So SK et al (2018) Disparities in hepatocellular carcinoma incidence by race/ethnicity and geographic area in California: implications for prevention. Cancer 124:3551-3559. https://doi.org/10.1002/cncr.31598 
36. Melkonian SC, Jim MA, Reilley B et al (2018) Incidence of primary liver cancer in American Indians and Alaska Natives, US, 1999-2009. Cancer Causes Control 29(9):833-844. https://doi. org/10.1007/s10552-018-1059-3

37. Sloane D, Chen H, Howell C (2006) Racial disparity in primary hepatocellular carcinoma: tumor stage at presentation, surgical treatment and survival. J Natl Med Assoc 98(12):1934-1939

38. Artinyan A, Mailey B, Sanchez-Luege N et al (2010) Race, ethnicity, and socioeconomic status influence the survival of patients with hepatocellular carcinoma in the United States. Cancer 116(5):1367-1377. https://doi.org/10.1002/cncr.24817

39. Njei B, Rotman Y, Ditah I, Lim JK (2015) Emerging trends in hepatocellular carcinoma incidence and mortality. Hepatology 61(1):191-199. https://doi.org/10.1002/hep.27388

40. Wong LL, Hernandez B, Kwee S, Albright CL, Okimoto G, Tsai N (2012) Healthcare disparities in Asians and Pacific Islanders with hepatocellular cancer. Am J Surg. https://doi.org/10.1016/j. amjsurg.2011.06.055

41. Cook MB, Dawsey SM, Freedman ND et al (2009) Sex disparities in cancer incidence by period and age. Cancer Epidemiol Biomark Prev. https://doi.org/10.1158/1055-9965.EPI-08-1118

42. Rahib L, Smith BD, Aizenberg R, Rosenzweig AB, Fleshman JM, Matrisian LM (2014) Projecting cancer incidence and deaths to 2030: the unexpected burden of thyroid, liver, and pancreas cancers in the United States. Cancer Res. https://doi. org/10.1158/0008-5472.CAN-14-0155

43. Yeh SH, Chen PJ (2010) Gender disparity of hepatocellular carcinoma: the roles of sex hormones. Oncology. https://doi. org/10.1159/000315247

44. Naugler WE, Sakurai T, Kim S et al (2007) Gender disparity in liver cancer due to sex differences in MyD88-dependent IL-6 production. Science 317(5834):121-124. https://doi.org/10.1126/ science. 1140485

45. National Cancer Institute. Race recode changes. https://seer.cance r.gov/seerstat/variables/seer/race_ethnicity/.

46. Boyle P, Parkin DM (1991) Cancer registration: principles and methods. Statistical methods for registries. IARC Sci Publ. 95:126-158

47. National Cancer Institute (2017) Bayesian Information Criterion (BIC) details. https://surveillance.cancer.gov/joinpoint/faq/bic_ details.html.

48. Schwarz G (1978) Estimating the dimension of a model. Ann Stat 6(2):461-464. https://doi.org/10.1214/aos/1176344136

49. Kim H-J, Fay MP, Feuer EJ, Midthune DN (1998) Permutation tests for joipoint regression with applications to cancer rates. Stat Med 2000(19):335-351. https://doi.org/10.1002/(SICI)10970258(20000215)19:33.3.CO;2-Q

50. National Cancer Institute (2017) Permutation test details. https:// surveillance.cancer.gov/joinpoint/faq/details.html.

51. Centers for Disease Control and Prevention (2017) National Program of Cancer Registries. https://www.cdc.gov/cancer/npcr/ about.htm. Accessed 8 Sept 2017

52. National Cancer Institute (2017) Cancer incidence rates adjusted for reporting delay. https://surveillance.cancer.gov/delay/. Accessed 2 Feb 2018

53. Holford TR, Zhang Z, McKay LA (1994) Estimating age, period and cohort effects using the multistage model for cancer. Stat Med 13(1):23-41. https://doi.org/10.1002/sim.4780130105

54. Armstrong GL, Wasley A, Simard EP, McQuillan GM, Kuhnert WL, Alter MJ (2006) The prevalence of hepatitis C virus infection in the United States, 1999 through 2002. Ann Intern Med 144(10):705-714

55. Smith BD, Morgan RL, Beckett GA et al (2012) Recommendations for the identification of chronic hepatitis $\mathrm{C}$ virus infection among persons born during 1945-1965. Morb Mortal Wkly Rep 61(4):1-32
56. Davis GL, Roberts WL (2010) The healthcare burden imposed by liver disease in aging baby boomers. Curr Gastroenterol Rep 12(1):1-6. https://doi.org/10.1007/s 11894-009-0087-2

57. Kim H-J, Fay M, Feuer EJ, Midthune DN (2000) Permutation tests for jointpoint regression with applications to cancer rates. Stat Med 19:335-351. https://doi.org/10.1002/(SICI)10970258(20000215)19:33.3.CO;2-Q

58. Hudson DJ (1966) Fitting segmented curves whose join points have to be estimated. J Am Stat Assoc 61(316):1097-1129

59. Kim H-J, Yu B, Feuer EJ (2008) Inference in segmented line regression: a simulation study. J Stat Comput Simul 78(11):1087-1103. https://doi.org/10.1080/009496507015284 61

60. Kim H-J, Fay MP, Yu B, Barrett MJ, Feuer EJ (2004) Comparability of segmented line regression models. Biometrics 60(4):10051014. https://doi.org/10.1111/j.0006-341X.2004.00256.x

61. National Cancer Institute. Joinpoint: frequently asked questions. https://surveillance.cancer.gov/joinpoint/faq/. Accessed 14 Apr 2014.

62. Kim H-J, Yu B, Feuer EJ. Selection the number of change-points in segmented line regression. https://www.ncbi.nlm.nih.gov/pmc/ articles/PMC2737518/pdf/nihms134673.pdf. Accessed 15 Apr 2017.

63. Siegel RL, Miller KD, Jemal A (2016) Cancer statistics, 2016. CA Cancer J Clin 66(1):7-30. https://doi.org/10.3322/caac.21332

64. National Cancer Institute (2017) Cancer Health Disparities Research. https://www.cancer.gov/research/areas/disparities. Accessed 14 Apr 2017

65. Hausauer AK, Keegan TH, Chang ET, Clarke CA, Org H (2007) Recent breast cancer trends among Asian/Pacific Islander, Hispanic, and African-American women in the US: changes by tumor subtype. Breast Cancer Res 9:R90. https://doi.org/10.1186/bcr18 39

66. Cress RD, Morris C, Ellison GL, Goodman MT (2006) Secular changes in colorectal cancer incidence by subsite, stage at diagnosis, and race/ethnicity, 1992-2001. Cancer 107(S5):1142-1152. https://doi.org/10.1002/cncr.22011

67. Ward EM, Sherman RL, Henley SJ et al (2019) Annual report to the nation on the status of cancer, featuring cancer in men and women age 20-49 years. JNCI J Natl Cancer Inst 111:1-19. https ://doi.org/10.1093/jnci/djz106

68. Siegel RL, Miller KD, Jemal A (2019) Cancer statistics, 2019. CA Cancer J Clin 69(1):7-34. https://doi.org/10.3322/caac.21551

69. Donato F, Boffetta P, Puoti M (1998) A meta-analysis of epidemiological studies on the combined effect of hepatitis $\mathrm{B}$ and $\mathrm{C}$ virus infections in causing hepatocellular carcinoma. Int J Cancer 75(3):347-354. https://doi.org/10.1002/(SICI)1097-0215(19980 130)75:3\%3c347:AID-IJC4\%3e3.0.CO;2-2

70. Ditah I, Ditah F, Devaki P et al (2014) The changing epidemiology of hepatitis $C$ virus infection in the United States: National health and nutrition examination survey 2001 through 2010. J Hepatol 60(4):691-698. https://doi.org/10.1016/j.jhep.2013.11.014

71. Mitchell T, Armstrong GL, Hu DJ, Wasley A, Painter JA (2011) The increasing burden of imported chronic hepatitis BUnited States, 1974-2008. PLoS ONE 6(12):6-11. https://doi. org/10.1371/journal.pone.0027717

72. Kim WR (2012) Epidemiology of hepatitis B in the United States. Hepatology 49(3):S28-S34. https://doi.org/10.1002/hep.22975 .Epidemiology

73. Chuang S-C, Lee Y-CA, Wu G-J, Straif K, Hashibe M (2015) Alcohol consumption and liver cancer risk: a meta-analysis. Cancer Causes Control 26(9):1205-1231. https://doi.org/10.1007/ s10552-015-0615-3

74. Greenfield TK, Kerr WC (2003) Tracking alcohol consumption over time. https://pubs.niaaa.nih.gov/publications/arh27-1/30-38. htm 
75. Haughwout SP, Slater ME (2017) Apparent per capita alcohol consumption: national, state, and regional trends, 1977-2015.

76. Centers for Disease Control and Prevention (2016) Trends in current cigarette smoking among high school students and adults, United States, 1965-2014. https://www.cdc.gov/tobacco/data statistics/tables/trends/cig_smoking/. Accessed 4 Apr 2017

77. Lee YCA, Cohet C, Yang YC, Stayner L, Hashibe M, Straif K (2009) Meta-analysis of epidemiologic studies on cigarette smoking and liver cancer. Int J Epidemiol. https://doi.org/10.1093/ ije/dyp280

Publisher's Note Springer Nature remains neutral with regard to jurisdictional claims in published maps and institutional affiliations. 